Marine Biology 29, 15-28 (1975)

(C) by Springer-Verlag 1975

\title{
The Breeding Biology of two Sympatric Species of Tropical Intertidal Hermit Crabs, Clibanarius chapini and $C$. senegalensis
}

\author{
C. Ameyaw-Akumfi \\ Department of Zoology, University of Cape Coast; Cape Coast, Ghana
}

\section{Abstract}

A one-year study of breeding in the two intertidal hermit crabs Clibanarius chapini schmitt and $C$. senegalensis Chevreux and Bouvier showed that both species maintain a high reproductive activity throughout the year. Brief low reproductive activities, found in both species, occurred in different months: $C$. chapini in November, $C$. senegalensis in May. While these drops cannot be attributed to any recognisable environmental factor, it is suggested that such pattern may result from competition between the two species. Sex-ratio studies indicated slightly more females than males in both species. The maximal size attained by males of both species is considerably greater than that of the females; in $C$. senegalensis this is very marked. It is suggested that it may be selectively advantageous for a female to attain a smaller maximal size since her brood must also be accomodated within the shell. As expected for continuously breeding species, recruitment of young into the population is also continuous.

\section{Introduction}

There has been considerable interest in the reproductive cycles of tropical marine invertebrates with regard to some environmental factors. Thorson (1946, 1950), Gunter (1957), Moore (1958) and Giese (1959) emphasize temperature as an important controlling factor. Sandoz and Rogers (1944), Knight (1967), and Buchanan and Milleman (1969) consider the effects of salinity, while knowlton (1965) shows the importance of food. However, complete studies of breeding cycles of marine invertebrates have been fragmentary (Goodbody, 1965).

Among crustaceans, continuous breeding cycles have been reported in Puggetia producta (Boolootian et al., 1959), Mysidium columbiae and Emerita portoricensis (Goodbody, 1965), Petrolisthes ezongatus (Greenwood, 1965), Calcinus laevimanus and C. Zatens (Reese, 1968) - both with brief quiescent periods, and Clibanarius zebra (Reese, 1968). As will be brought up later, all these species show breeding peaks and it is questionable as to the relevant trigger or triggers from the environment which might be involved.
Among the non-continuous breeders, Boolootian et $a$. (1959) provide data on Pachygrapsus crassipes and Emerita analoga from the Californian coast. Palaemonetes pugio is a summer breeder (Little, 1968), so is Palaemon macrodactylus (Litt1e, 1969). Uca pugizator breeds actively between May and October (Herrnkind, 1968). Carcinus tibicens and Clibanarius tricotor from Barbados, West Indies, are both seasonal breeders (Lewis, 1960).

In this work, the breeding biology of two species of Clibanamius, $C$. chapini and $C$. senegalensis, has been investigated to add to our knowledge in this area of crustacean biology.

\section{Material and Methods}

Hermit crabs were obtained at Tengpobo, a fishing village near Prampram (ca. $6 \mathrm{ON}^{\mathrm{N}}$ ), about. $60 \mathrm{~km}$ to the east of Accra, Ghana. Two-meter quadrat samples were collected from late September, 1969 to June, 1970. Thereafter, non-quantitative observations were made up to September, 1970 so as to complete a whole year of observations. The collected crabs were taken to the Department of Zoology, University of Ghana, Legon. Various methods employed are described under each subtopic.

\section{Obsemations}

Breeding Period

Throughout the sampling period, ovigerous females of both species were present in all samples. During the first 6 weeks, sampling was done weekly; thereafter, samples were taken once a fortnight or once a month.

Tables 1 and 2 show the numbers of female crabs in berry in each sample; places and dates of sampling are also given.

Fig. 1 illustrates the percentages of females in berry in monthly samples. In both species there was a consistently high percentage of females in berry throughout the period of sampling, except for a sharp drop in the case of Clibanarius chapini in November, 1969 and in C. senegalensis in May, 1970 . 
Table 1. Clibonamius chopini. Percentage females in berry and those with and without ovarian eggs collected at Tengpobo, Ghana

\begin{tabular}{llllllll}
\hline Date & Distance & No. of & No. & \% total & No. & $\%$ total & $\%$ total \\
from MLT & females with & with & in & in ber- & with \\
mark; & collect- & eggs & eggs & berry & ry & ovarian \\
seaward & ed & & & & & eggs \\
(m) & & & & & only
\end{tabular}

\section{9}

$\begin{array}{rrrrrrrr}24 \text { Sept. } & 10 & 16 & 16 & 100.0 & 14 & 87.5 & 12.5 \\ 4 \text { Oct. } & 10 & 14 & 12 & 85.7 & 11 & 78.5 & 7.2 \\ 24 \text { Oct. } & 10 & 63 & 58 & 92.0 & 47 & 75.0 & 17.0 \\ & 100 & 137 & 109 & 79.5 & 82 & 60.0 & 19.5 \\ 31 \text { Oct. } & 50 & 2 & 2 & 100.0 & 2 & & \\ & \text { beyond } 100 & 60 & 58 & 96.0 & 57 & 95.0 & 1.0 \\ 12 \text { Nov. } & 10 & 71 & 69 & 97.2 & 29 & 41.0 & 56.2 \\ & 50 & 36 & 28 & 77.8 & 20 & 55.8 & 22.0 \\ & 100 & 18 & 10 & 55.6 & 4 & 22.3 & 33.3 \\ 28 \text { Nov. } & 10 & 21 & 12 & 57.1 & 5 & 24.0 & 33.1 \\ & 50 & 16 & 14 & 87.5 & 7 & 43.8 & 43.7 \\ 12 \text { Dec. } & 10 & 234 & 212 & 90.0 & 152 & 65.0 & 25.0 \\ & 50 & 64 & 41 & 64.0 & 29 & 45.4 & 18.6\end{array}$

1970

\begin{tabular}{|c|c|c|c|c|c|c|c|c|}
\hline 23 & Jan. & $\begin{array}{r}10 \\
50 \\
100\end{array}$ & $\begin{array}{r}78 \\
315 \\
106\end{array}$ & $\begin{array}{r}63 \\
275 \\
86\end{array}$ & $\begin{array}{l}81.0 \\
87.3 \\
81.0\end{array}$ & $\begin{array}{r}61 \\
252 \\
79\end{array}$ & $\begin{array}{l}78.2 \\
80.0 \\
75.0\end{array}$ & $\begin{array}{l}2.8 \\
7.3 \\
6.0\end{array}$ \\
\hline 11 & Feb. & $\begin{array}{r}10 \\
50 \\
100\end{array}$ & $\begin{array}{r}113 \\
40 \\
54\end{array}$ & $\begin{array}{r}108 \\
34 \\
53\end{array}$ & $\begin{array}{l}95.6 \\
85.0 \\
98.0\end{array}$ & $\begin{array}{l}49 \\
29 \\
53\end{array}$ & $\begin{array}{l}43.5 \\
72.5 \\
98.0\end{array}$ & $\begin{array}{l}52.1 \\
12.5 \\
98.0\end{array}$ \\
\hline 27 & Feb. & $\begin{array}{r}10 \\
50 \\
100\end{array}$ & $\begin{array}{l}76 \\
72 \\
54\end{array}$ & $\begin{array}{l}50 \\
67 \\
54\end{array}$ & $\begin{array}{r}65.8 \\
93.1 \\
100.0\end{array}$ & $\begin{array}{l}47 \\
63 \\
48\end{array}$ & $\begin{array}{l}62.0 \\
87.5 \\
88.9\end{array}$ & $\begin{array}{r}3.8 \\
5.6 \\
11.1\end{array}$ \\
\hline 20 & March & $\begin{array}{r}10 \\
50 \\
100\end{array}$ & $\begin{array}{r}121 \\
74 \\
42\end{array}$ & $\begin{array}{r}117 \\
70 \\
42\end{array}$ & $\begin{array}{r}96.7 \\
94.6 \\
100.0\end{array}$ & $\begin{array}{r}109 \\
66 \\
42\end{array}$ & $\begin{array}{r}90.1 \\
89.2 \\
100.0\end{array}$ & $\begin{array}{l}6.6 \\
5.4 \\
0.0\end{array}$ \\
\hline 20 & April & $\begin{array}{l}10 \\
50\end{array}$ & $\begin{array}{r}136 \\
70\end{array}$ & $\begin{array}{r}131 \\
67\end{array}$ & $\begin{array}{l}96.3 \\
95.7\end{array}$ & $\begin{array}{r}124 \\
66\end{array}$ & $\begin{array}{l}91.2 \\
94.3\end{array}$ & $\begin{array}{l}5.1 \\
1.4\end{array}$ \\
\hline 23 & May & $\begin{array}{l}10 \\
50\end{array}$ & $\begin{array}{r}29 \\
132\end{array}$ & $\begin{array}{r}26 \\
125\end{array}$ & $\begin{array}{l}89.5 \\
94.5\end{array}$ & $\begin{array}{r}23 \\
121\end{array}$ & $\begin{array}{l}79.5 \\
91.5\end{array}$ & $\begin{array}{r}10.0 \\
3.0\end{array}$ \\
\hline 20 & June & 10 & 152 & 139 & 91.4 & 130 & 85.5 & 5.9 \\
\hline
\end{tabular}

$\mathrm{a}_{10}, 50$ and $100 \mathrm{~m}$ are distances from MLT (mean low tide) mark, seaward, to points of collection. All collections were made at low tide, and the areas were covered by no more than $25 \mathrm{~cm}$ water. 
Table 2. Clibcharius senegalensis. Percentage females in berry and those with and without ovarian eggs collected at Tengpobo, Ghana

\begin{tabular}{|c|c|c|c|c|c|c|c|}
\hline Date & $\begin{array}{l}\text { Distance } \\
\text { from MLT } \\
\text { mark; } \\
\text { seaward } \\
\text { (m) }{ }^{a}\end{array}$ & $\begin{array}{l}\text { No. of } \\
\text { females } \\
\text { collect- } \\
\text { ed }\end{array}$ & $\begin{array}{l}\text { No. } \\
\text { with } \\
\text { eggs }\end{array}$ & $\begin{array}{l}\% \text { total } \\
\text { with } \\
\text { eggs }\end{array}$ & $\begin{array}{l}\text { No. } \\
\text { in } \\
\text { berry }\end{array}$ & $\begin{array}{l}\% \text { total } \\
\text { in ber- } \\
\text { ry }\end{array}$ & $\begin{array}{l}\% \text { total } \\
\text { with } \\
\text { ovarian } \\
\text { eggs } \\
\text { only }\end{array}$ \\
\hline \multicolumn{8}{|l|}{1969} \\
\hline 29 Sept. & 10 & 158 & 137 & 86.7 & 111 & 69.8 & 16.9 \\
\hline 4 Oct. & $\begin{array}{l}10 \\
50\end{array}$ & $\begin{array}{l}54 \\
32\end{array}$ & $\begin{array}{l}47 \\
27\end{array}$ & $\begin{array}{l}87.0 \\
84.5\end{array}$ & $\begin{array}{l}30 \\
22\end{array}$ & $\begin{array}{l}55.8 \\
69.0\end{array}$ & $\begin{array}{l}31.2 \\
15.5\end{array}$ \\
\hline 15 oct. & 10 & 75 & 66 & 88.0 & 46 & 61.3 & 26.7 \\
\hline 24 Oct. & $\begin{array}{r}10 \\
50 \\
100\end{array}$ & $\begin{array}{r}320 \\
26 \\
9\end{array}$ & $\begin{array}{r}280 \\
19 \\
8\end{array}$ & $\begin{array}{l}88.5 \\
73.0 \\
89.0\end{array}$ & $\begin{array}{r}250 \\
16 \\
4\end{array}$ & $\begin{array}{l}78.0 \\
61.6 \\
44.5\end{array}$ & $\begin{array}{l}10.5 \\
11.4 \\
44.5\end{array}$ \\
\hline 31 Oct. & $\begin{array}{l}10 \\
50 \\
100 \\
\text { beyond } 100\end{array}$ & $\begin{array}{l}61 \\
67 \\
54 \\
19\end{array}$ & $\begin{array}{l}53 \\
60 \\
48 \\
15\end{array}$ & $\begin{array}{l}87.0 \\
90.0 \\
89.0 \\
79.0\end{array}$ & $\begin{array}{l}34 \\
45 \\
41 \\
15\end{array}$ & $\begin{array}{l}56.0 \\
67.3 \\
76.0 \\
79.0\end{array}$ & $\begin{array}{r}31.0 \\
22.7 \\
13.0 \\
0.0\end{array}$ \\
\hline 12 Nov. & $\begin{array}{r}10 \\
50 \\
100\end{array}$ & $\begin{array}{r}7 \\
34 \\
2\end{array}$ & $\begin{array}{r}6 \\
31 \\
2\end{array}$ & $\begin{array}{r}85.8 \\
91.2 \\
100.0\end{array}$ & $\begin{array}{r}5 \\
25\end{array}$ & $\begin{array}{l}71.5 \\
73.5\end{array}$ & $\begin{array}{l}14.3 \\
17.7\end{array}$ \\
\hline 28 Nov. & $\begin{array}{l}10 \\
50\end{array}$ & $\begin{array}{r}53 \\
301\end{array}$ & $\begin{array}{r}37 \\
281\end{array}$ & $\begin{array}{l}69.7 \\
93.3\end{array}$ & $\begin{array}{r}25 \\
208\end{array}$ & $\begin{array}{l}47.3 \\
69.0\end{array}$ & $\begin{array}{l}22.4 \\
24.3\end{array}$ \\
\hline 12 Dec. & 10 & 125 & 92 & 73.8 & 72 & 57.8 & 16.0 \\
\hline \multicolumn{8}{|l|}{1970} \\
\hline $23 \mathrm{Jan}$. & $\begin{array}{r}10 \\
50 \\
100\end{array}$ & $\begin{array}{r}96 \\
200 \\
21\end{array}$ & $\begin{array}{r}81 \\
173 \\
8\end{array}$ & $\begin{array}{l}84.3 \\
86.5 \\
38.2\end{array}$ & $\begin{array}{r}77 \\
162 \\
5\end{array}$ & $\begin{array}{l}80.0 \\
81.0 \\
23.8\end{array}$ & $\begin{array}{r}4.3 \\
5.5 \\
14.4\end{array}$ \\
\hline 11 Feb. & $\begin{array}{r}10 \\
50 \\
100\end{array}$ & $\begin{array}{l}44 \\
39 \\
84\end{array}$ & $\begin{array}{l}39 \\
35 \\
82\end{array}$ & $\begin{array}{l}88.6 \\
90.0 \\
98.0\end{array}$ & $\begin{array}{l}32 \\
29 \\
82\end{array}$ & $\begin{array}{l}73.0 \\
74.5 \\
98.0\end{array}$ & $\begin{array}{r}15.6 \\
25.5 \\
0.0\end{array}$ \\
\hline 27 Feb. & $\begin{array}{r}10 \\
50 \\
100\end{array}$ & $\begin{array}{r}104 \\
22 \\
64\end{array}$ & $\begin{array}{l}80 \\
21 \\
62\end{array}$ & $\begin{array}{l}76.9 \\
95.5 \\
96.9\end{array}$ & $\begin{array}{l}68 \\
18 \\
58\end{array}$ & $\begin{array}{l}86.4 \\
81.8 \\
90.6\end{array}$ & $\begin{array}{r}11.5 \\
13.7 \\
6.3\end{array}$ \\
\hline 20 March & $\begin{array}{r}10 \\
50 \\
100\end{array}$ & $\begin{array}{l}50 \\
28 \\
87\end{array}$ & $\begin{array}{l}46 \\
24 \\
84\end{array}$ & $\begin{array}{l}92.0 \\
85.7 \\
96.6\end{array}$ & $\begin{array}{l}45 \\
23 \\
77\end{array}$ & $\begin{array}{l}90.1 \\
82.1 \\
88.5\end{array}$ & $\begin{array}{l}1.9 \\
2.6 \\
8.1\end{array}$ \\
\hline 20 ApriI & $\begin{array}{l}10 \\
50\end{array}$ & $\begin{array}{l}70 \\
73\end{array}$ & $\begin{array}{l}58 \\
68\end{array}$ & $\begin{array}{l}82.9 \\
93.2\end{array}$ & $\begin{array}{l}55 \\
63\end{array}$ & $\begin{array}{l}78.6 \\
86.3\end{array}$ & $\begin{array}{l}4.3 \\
6.9\end{array}$ \\
\hline 23 May & $\begin{array}{l}10 \\
50\end{array}$ & $\begin{array}{l}161 \\
168\end{array}$ & $\begin{array}{l}111 \\
162\end{array}$ & $\begin{array}{l}69.0 \\
96.5\end{array}$ & $\begin{array}{l}63 \\
82\end{array}$ & $\begin{array}{l}39.0 \\
48.8\end{array}$ & $\begin{array}{l}30.0 \\
47.7\end{array}$ \\
\hline 20 June & 10 & 95 & 89 & 93.7 & 64 & 67.4 & 26.3 \\
\hline
\end{tabular}

$\mathrm{a}_{10}, 50$ and $100 \mathrm{~m}$ are distances from MLT mark, seaward, to points of collection. All collections were made at low tide, and the areas were covered by no more than $25 \mathrm{~cm}$ water. 
Table 3. Clibanarius chapini and $C$. senegalensis. Total numbers of females with single and double batches of embryonic stages

\begin{tabular}{|c|c|c|c|c|c|c|c|c|c|c|c|}
\hline \multirow[t]{3}{*}{ Date } & \multirow{3}{*}{$\begin{array}{l}\text { Distance } \\
\text { from MLT } \\
\text { mark; } \\
\text { seaward } \\
\text { (m) }\end{array}$} & \multicolumn{5}{|c|}{ c. chapini } & \multicolumn{5}{|c|}{ C. senegalensis } \\
\hline & & \multirow{2}{*}{$\begin{array}{l}\text { No. } \\
\text { with } \\
\text { eggs }\end{array}$} & \multicolumn{2}{|c|}{$\begin{array}{l}\text { No. with } \\
1 \text { batch }\end{array}$} & \multicolumn{2}{|c|}{$\begin{array}{l}\text { No. with } \\
2 \text { batches }\end{array}$} & \multirow{2}{*}{$\begin{array}{l}\text { No. } \\
\text { with } \\
\text { eggs }\end{array}$} & \multicolumn{2}{|c|}{$\begin{array}{l}\text { No, with } \\
1 \text { batch }\end{array}$} & \multicolumn{2}{|c|}{$\begin{array}{l}\text { No. with } \\
2 \text { batches }\end{array}$} \\
\hline & & & No. & $\begin{array}{l}\% \\
\text { total }\end{array}$ & No. & $\begin{array}{l}\% \\
\text { tota } 1\end{array}$ & & No. & $\begin{array}{l}\% \\
\text { total }\end{array}$ & No. & $\begin{array}{l}\% \\
\text { total }\end{array}$ \\
\hline \multicolumn{12}{|l|}{1969} \\
\hline 29 Sept. & 10 & 16 & 2 & 12.5 & 14 & 87.5 & 137 & 26 & 18.0 & 111 & 82.0 \\
\hline 4 Oct. & $\begin{array}{l}10 \\
50\end{array}$ & 12 & 1 & 8.3 & 10 & 91.7 & $\begin{array}{l}47 \\
27\end{array}$ & $\begin{array}{r}23 \\
6\end{array}$ & $\begin{array}{l}49.0 \\
22.3\end{array}$ & $\begin{array}{l}24 \\
21\end{array}$ & $\begin{array}{l}51.0 \\
77.7\end{array}$ \\
\hline 24 oct. & $\begin{array}{r}10 \\
50 \\
100\end{array}$ & $\begin{array}{r}58 \\
9 \\
109\end{array}$ & $\begin{array}{r}11 \\
4 \\
27\end{array}$ & $\begin{array}{l}19.0 \\
44.5 \\
24.8\end{array}$ & $\begin{array}{r}47 \\
5 \\
82\end{array}$ & $\begin{array}{l}81.0 \\
55.5 \\
75.2\end{array}$ & $\begin{array}{r}280 \\
19 \\
9\end{array}$ & $\begin{array}{r}30 \\
5 \\
4\end{array}$ & $\begin{array}{l}10.8 \\
26.4 \\
44.5\end{array}$ & $\begin{array}{r}250 \\
14 \\
5\end{array}$ & $\begin{array}{l}89.2 \\
73.6 \\
55.5\end{array}$ \\
\hline 31 oct. & $\begin{array}{l}10 \\
50 \\
100 \\
\text { beyond } 100\end{array}$ & 58 & 57 & 98.5 & 1 & 1.5 & $\begin{array}{l}53 \\
60 \\
48 \\
15\end{array}$ & $\begin{array}{r}20 \\
27 \\
9 \\
3\end{array}$ & $\begin{array}{l}37.8 \\
45.0 \\
18.8 \\
20.0\end{array}$ & $\begin{array}{l}33 \\
33 \\
39 \\
12\end{array}$ & $\begin{array}{l}62.2 \\
55.0 \\
81.2 \\
80.0\end{array}$ \\
\hline 12 Nov. & $\begin{array}{r}10 \\
50 \\
100\end{array}$ & $\begin{array}{l}69 \\
28 \\
10\end{array}$ & $\begin{array}{r}42 \\
8 \\
6\end{array}$ & $\begin{array}{l}61.0 \\
28.5 \\
60.0\end{array}$ & $\begin{array}{r}27 \\
20 \\
4\end{array}$ & $\begin{array}{l}39.0 \\
71.5 \\
40.0\end{array}$ & $\begin{array}{r}6 \\
31\end{array}$ & $\begin{array}{l}2 \\
9\end{array}$ & $\begin{array}{l}33.3 \\
29.0\end{array}$ & $\begin{array}{r}4 \\
22\end{array}$ & $\begin{array}{l}66.7 \\
71.0\end{array}$ \\
\hline 28 Nov. & $\begin{array}{l}10 \\
50\end{array}$ & $\begin{array}{l}12 \\
14\end{array}$ & $\begin{array}{r}11 \\
7\end{array}$ & $\begin{array}{l}92.0 \\
50.0\end{array}$ & $\frac{1}{7}$ & $\begin{array}{r}8.0 \\
50.0\end{array}$ & $\begin{array}{r}37 \\
281\end{array}$ & $\begin{array}{l}25 \\
90\end{array}$ & $\begin{array}{l}67.5 \\
32.0\end{array}$ & $\begin{array}{r}12 \\
191\end{array}$ & $\begin{array}{l}32.5 \\
68.0\end{array}$ \\
\hline $12 \mathrm{Dec}$ & $\begin{array}{l}10 \\
50\end{array}$ & $\begin{array}{r}212 \\
41\end{array}$ & $\begin{array}{l}78 \\
18\end{array}$ & $\begin{array}{l}36.7 \\
44.0\end{array}$ & $\begin{array}{r}134 \\
23\end{array}$ & $\begin{array}{l}63.3 \\
56.0\end{array}$ & 92 & 27 & 29.4 & 65 & 70.6 \\
\hline
\end{tabular}

1970

\begin{tabular}{|c|c|c|c|c|c|c|c|c|c|c|c|c|}
\hline 23 & Jan. & $\begin{array}{r}10 \\
50 \\
100\end{array}$ & $\begin{array}{r}63 \\
275 \\
84\end{array}$ & $\begin{array}{r}8 \\
25 \\
9\end{array}$ & $\begin{array}{r}12.7 \\
9.1 \\
10.5\end{array}$ & $\begin{array}{r}55 \\
250 \\
75\end{array}$ & $\begin{array}{l}87.3 \\
90.9 \\
89.5\end{array}$ & $\begin{array}{r}81 \\
173 \\
8\end{array}$ & $\begin{array}{r}4 \\
11 \\
3\end{array}$ & $\begin{array}{r}5.0 \\
6.4 \\
37.5\end{array}$ & $\begin{array}{r}77 \\
164 \\
5\end{array}$ & $\begin{array}{l}95.0 \\
93.6 \\
62.5\end{array}$ \\
\hline 11 & Feb. & $\begin{array}{r}10 \\
50 \\
100\end{array}$ & $\begin{array}{r}108 \\
34 \\
53\end{array}$ & $\begin{array}{r}78 \\
5 \\
15\end{array}$ & $\begin{array}{l}72.2 \\
14.7 \\
28.3\end{array}$ & $\begin{array}{l}30 \\
29 \\
38\end{array}$ & $\begin{array}{l}27.8 \\
85.3 \\
71.7\end{array}$ & $\begin{array}{l}39 \\
35 \\
82\end{array}$ & $\begin{array}{l}17 \\
18 \\
28\end{array}$ & $\begin{array}{l}43.6 \\
51.4 \\
34.1\end{array}$ & $\begin{array}{l}22 \\
17 \\
54\end{array}$ & $\begin{array}{l}56.4 \\
48.6 \\
65.9\end{array}$ \\
\hline 27 & Feb. & $\begin{array}{r}10 \\
50 \\
100\end{array}$ & $\begin{array}{l}50 \\
67 \\
54\end{array}$ & $\begin{array}{r}11 \\
15 \\
7\end{array}$ & $\begin{array}{l}22.0 \\
22.4 \\
13.0\end{array}$ & $\begin{array}{l}39 \\
52 \\
47\end{array}$ & $\begin{array}{l}78.0 \\
77.6 \\
87.0\end{array}$ & $\begin{array}{l}80 \\
21 \\
62\end{array}$ & $\begin{array}{r}14 \\
4 \\
7\end{array}$ & $\begin{array}{l}17.5 \\
19.0 \\
11.3\end{array}$ & $\begin{array}{l}66 \\
17 \\
55\end{array}$ & $\begin{array}{l}82.5 \\
81.0 \\
88.7\end{array}$ \\
\hline 20 & March & $\begin{array}{r}10 \\
50 \\
100\end{array}$ & $\begin{array}{r}117 \\
70 \\
42\end{array}$ & $\begin{array}{r}10 \\
4 \\
0\end{array}$ & $\begin{array}{l}8.5 \\
5.7 \\
0.0\end{array}$ & $\begin{array}{r}107 \\
66 \\
42\end{array}$ & $\begin{array}{r}91.5 \\
94.7 \\
100.0\end{array}$ & $\begin{array}{l}46 \\
24 \\
84\end{array}$ & $\begin{array}{l}2 \\
1 \\
9\end{array}$ & $\begin{array}{r}4.3 \\
4.2 \\
10.7\end{array}$ & $\begin{array}{l}44 \\
23 \\
75\end{array}$ & $\begin{array}{l}95.7 \\
95.8 \\
89.3\end{array}$ \\
\hline 20 & April & $\begin{array}{l}10 \\
50\end{array}$ & $\begin{array}{r}131 \\
67\end{array}$ & $\begin{array}{r}21 \\
3\end{array}$ & $\begin{array}{r}16.0 \\
4.5\end{array}$ & $\begin{array}{r}110 \\
64\end{array}$ & $\begin{array}{l}84.0 \\
95.5\end{array}$ & $\begin{array}{l}58 \\
68\end{array}$ & $\begin{array}{l}4 \\
7\end{array}$ & $\begin{array}{r}6.9 \\
10.3\end{array}$ & $\begin{array}{l}54 \\
61\end{array}$ & $\begin{array}{l}93.1 \\
89.7\end{array}$ \\
\hline 23 & May & $\begin{array}{l}10 \\
50\end{array}$ & $\begin{array}{r}26 \\
125\end{array}$ & $\begin{array}{l}3 \\
6\end{array}$ & $\begin{array}{r}11.5 \\
4.8\end{array}$ & $\begin{array}{r}23 \\
119\end{array}$ & $\begin{array}{l}88.5 \\
95.2\end{array}$ & $\begin{array}{l}111 \\
162\end{array}$ & $\begin{array}{l}50 \\
90\end{array}$ & $\begin{array}{l}43.5 \\
55.5\end{array}$ & $\begin{array}{l}61 \\
72\end{array}$ & $\begin{array}{l}56.5 \\
44.5\end{array}$ \\
\hline 20 & June & 10 & 139 & 14 & 10.1 & 125 & 89.9 & 89 & 28 & 31.5 & 61 & 68.5 \\
\hline
\end{tabular}




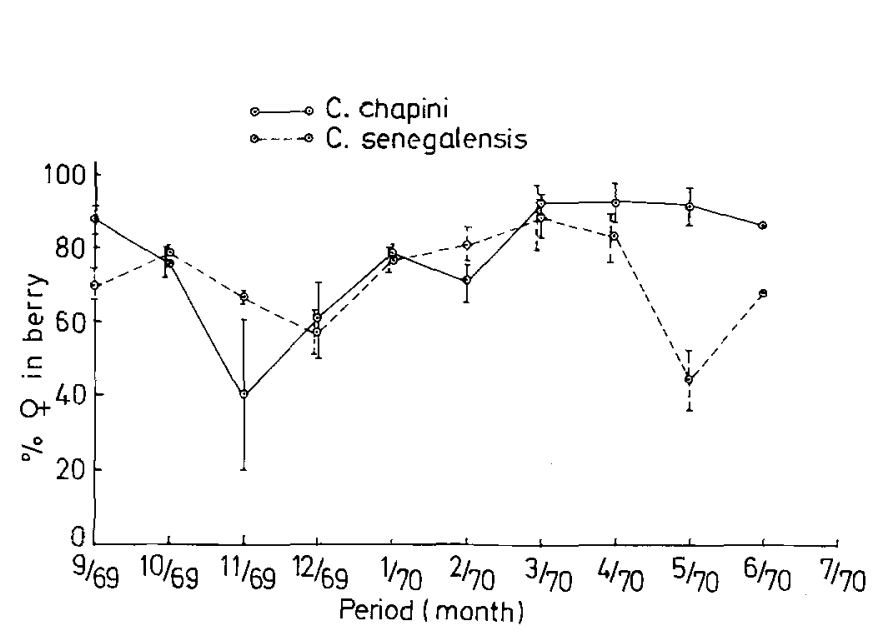

Fig. 1. Clibonarius spp. Percentages of females in berry in monthly samples, 1969 and 1970
C. chapini

$\leftrightarrow \rightarrow C$. senegalensis

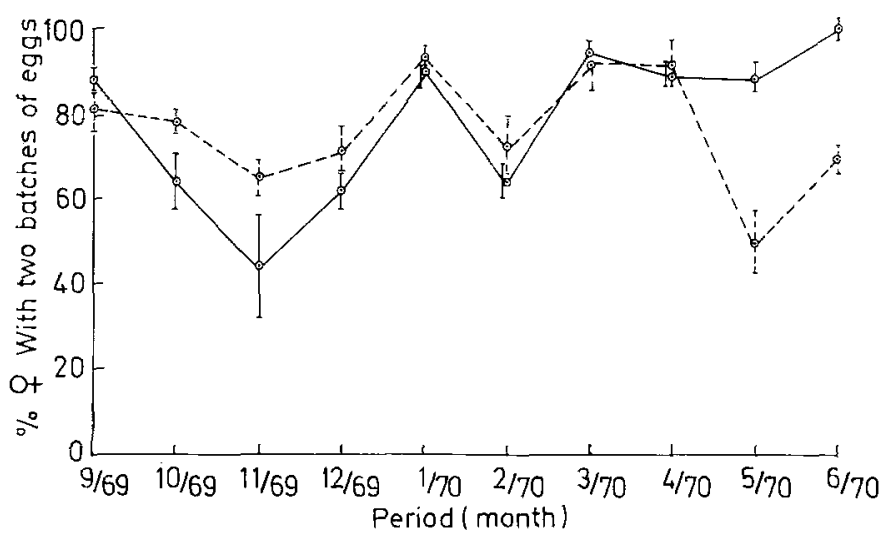

Fig. 2. Ciibanarius spp. Total numbers of females with two batches of embryonic stages in monthly samples, 1969 and 1970
A single female may have two batches of embryonic stages at the same time, one batch developing oocytes in the ovary, and the second embryos (dividing eggs or larvae) on the 3 abdominal appendages. Table 3 shows the percentages of females with one batch and those with two batches of embryonic stages. In Fig. 2 the percentages of females with two batches of embryonic stages in monthly samples are illustrated. Most females had two batches of embryonic stages. Batch ratio reflects the drops in percentage females in berry recorded in November, 1969 and in May, 1970 for Clibanamius chapini and $C$. senegalensis, respectively. Both species breed throughout the year.

\section{Fecundity}

As indicated previously, a single female may possess two batches of embryonic stages, one in the ovary and the other on the pleopods. In egg counts, the two batches were considered separately. Crabs in berry were carefully removed from their shells after being narcotized to prevent loss of eggs or larvae from their appendages; $5-15$ crabs of each species were used from each sample. The ovaries were dissected out, and the eggs separated and counted in small quantities $(\approx 100)$, using a dissecting microscope. The appendages to which the eggs and larvae were attached were cut of $f$, and the eggs or larvae separated.

In Table 4, the numbers of eggs in the ovary and on the abdominal appendages of individual females are shown. From this table, the following conclusions can be drawn: (1) Within the same size group there is considerable variation in the total number of eggs produced; (2) for each individual, the number of eggs in the ovary may differ marked1y from the number on the abdominal appendages; (3) fecundity is consistently high throughout the year.
Egg Size

Measurements were made of the diameter of ovarian and pleopodal eggs. Three females of each species were used and 200 eggs we re considered from each area. Fig. 3 shows typical histogram plots. A population of eggs from each area had about the same size, indicating that eggs from each location are most probably of the same age.

\section{Mating Behaviour and Mode of Fertilization}

Mating behaviour was observed twice in Clibanamis chapini and once in $C$. senegalensis. I could see no obvious differences between the two species. The following series of events were observed: Through some unknown mechanism, the male recognises the female (Hazlett, 1970, 1972, in a more detailed treatment of this topic, discusses the visua1, chemical and behavioural orientational components involved.) The male faced the female and gripped the aperture of the female's shell with his ambulatory legs. He then pushed his che1ipeds into the shell of the female, touching her legs in so doing. Then, at regular intervals, the male made pecking movements at the legs of the female with his chelipeds. He did not however grasp the female's legs. The pecking movement was periodically interrupted by a "shell-rocking" pattern. The male withdrew his chelipeds to grasp the edge of the female's shell. He then flexed and extended his chelipeds symmetrically so as to move the female's shell backwards and forwards. During this process a nubile female suddenly emerges from her shell as also does the male, and the two make ventral contacts for a few seconds. This is the copulatory act and, at the end of it, spermatophores are left at the genital openings of the female. The times recorded for this act for the three observations was between 4 and $7 \mathrm{sec}$. The basic pat- 
Table 4. Clibconarius chapini and C. senegalensis. Numbers of eggs or larvae on abdominal appendages and numbers of eggs in ovaries from individuals of various size groups

\begin{tabular}{|c|c|c|c|c|c|c|c|}
\hline \multirow[t]{2}{*}{ 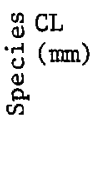 } & \multicolumn{4}{|c|}{$\begin{array}{l}\text { No. of eggs / larvae } \\
\text { on abdominal ap- } \\
\text { pendages }{ }^{a}\end{array}$} & \multirow[t]{2}{*}{$\begin{array}{l}\text { Embryonic } \\
\text { stage }\end{array}$} & \multirow{2}{*}{$\begin{array}{l}\text { No. of } \\
\text { ovar- } \\
\text { ian } \\
\text { eggsb }\end{array}$} & \multirow[t]{2}{*}{ Total } \\
\hline & 1 & 2 & 3 & Total & & & \\
\hline
\end{tabular}

3 Oct. 1969

\begin{tabular}{|c|c|c|c|c|c|c|}
\hline 4.0 & $\mathrm{~ns}$ & ns & ns & 449 & old eggs & None \\
\hline 5.0 & ns & ns & ns & 403 & old eggs & None \\
\hline 5.0 & 301 & 311 & 269 & 881 & $\begin{array}{l}\text { Young } \\
\text { larvae }\end{array}$ & None \\
\hline 先 & 138 & 122 & 94 & 354 & $\begin{array}{l}\text { Eggs about } \\
\text { to hatch }\end{array}$ & None \\
\hline 5.0 & 99 & 170 & 84 & 353 & $\begin{array}{l}\text { Eggs about } \\
\text { to hatch }\end{array}$ & 313 \\
\hline 5.0 & 128 & 210 & 99 & 437 & 01d eggs & 140 \\
\hline 5.0 & 163 & 277 & 224 & 664 & old eggs & $\mathrm{nc}$ \\
\hline 6.0 & ns & ns & ns & 495 & $\begin{array}{l}\text { Larvae, } \\
\text { just } \\
\text { hatched }\end{array}$ & 889 \\
\hline
\end{tabular}

\begin{tabular}{|c|c|c|c|c|c|c|c|}
\hline 4.5 & ns & ns & ns & 624 & old eggs & 712 & 1336 \\
\hline 5.0 & ns & ns & ns & 439 & $\begin{array}{l}\text { Larvae, } \\
\text { just } \\
\text { hatched }\end{array}$ & 910 & 1349 \\
\hline 5.0 & 178 & 287 & 90 & 555 & $\begin{array}{l}\text { Eggs about } \\
\text { to hatch }\end{array}$ & 1004 & 1559 \\
\hline $\ln _{5} 5.0$ & 137 & 287 & 224 & 648 & $\begin{array}{l}\text { Eggs about } \\
\text { to hatch }\end{array}$ & 580 & 1228 \\
\hline$\stackrel{\vec{\Phi}}{\stackrel{\sigma}{\sigma}} 5.0$ & 224 & 352 & 146 & 722 & old eggs & $\mathrm{nc}$ & 722 \\
\hline$\$ 5.5$ & 237 & 280 & 181 & 698 & Old eggs & 604 & 1302 \\
\hline $\begin{array}{l}\tilde{\Phi} 5.5 \\
\dot{0}\end{array}$ & ns & ns & ns & 479 & $\begin{array}{l}\text { Larvae, } \\
\text { just } \\
\text { hatched }\end{array}$ & 549 & 1028 \\
\hline 6.0 & $\mathrm{~ns}$ & ns & ns & 1182 & old eggs & 712 & 1894 \\
\hline 6.0 & 85 & 269 & 148 & 502 & $\begin{array}{l}\text { Eggs about } \\
\text { to hatch }\end{array}$ & $\mathrm{nc}$ & $502+$ \\
\hline 6.0 & $\mathrm{~ns}$ & ns & $\mathrm{ns}$ & 614 & $\begin{array}{l}\text { Larvae, } \\
\text { just } \\
\text { hatched }\end{array}$ & 305 & 919 \\
\hline
\end{tabular}

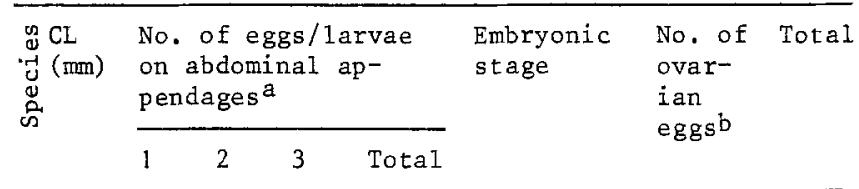

28 Nov. 1969

\begin{tabular}{|c|c|c|c|c|c|c|c|}
\hline 5.0 & 273 & 278 & 138 & 689 & Old eggs & $\mathrm{nc}$ & $689+$ \\
\hline . 5.5 & 176 & 187 & 103 & 466 & $\begin{array}{l}\text { Hatched } \\
\text { larvae }\end{array}$ & $\mathrm{nc}$ & $466+$ \\
\hline 5.5 & 166 & 178 & 91 & 435 & old eggs & $n c$ & $435+$ \\
\hline 5.5 & ns & ns & ns & 627 & 01d eggs & $\mathrm{nc}$ & $627+$ \\
\hline 5.5 & 202 & 241 & 139 & 582 & $01 \mathrm{~d}$ eggs & $n c$ & $582+$ \\
\hline 5.5 & ns & ns & ns & 299 & $\begin{array}{l}\text { Hatched } \\
\text { larvae }\end{array}$ & 319 & 618 \\
\hline
\end{tabular}

\begin{tabular}{|c|c|c|c|c|c|c|c|}
\hline 3.0 & ns & ns & ns & 166 & $\begin{array}{l}\text { Hatched } \\
\text { larvae }\end{array}$ & 135 & 301 \\
\hline 4.5 & 195 & 271 & 182 & 648 & old eggs & ne & $648+$ \\
\hline 4.5 & 192 & 240 & 132 & 564 & Old eggs & nc & $564+$ \\
\hline 4.5 & 271 & 334 & 228 & 833 & 01d eggs & nc & $833+$ \\
\hline क्ष 5.0 & 153 & 149 & 48 & 350 & old eggs & 274 & 624 \\
\hline 85.0 & 127 & 154 & 60 & 341 & $\begin{array}{l}\text { About to } \\
\text { hatch }\end{array}$ & 682 & 1023 \\
\hline 5.5 & ns & ns & ns & 654 & $\begin{array}{l}\text { Hatched } \\
\text { larvae }\end{array}$ & 497 & 1148 \\
\hline 5.5 & 454 & 596 & 434 & 1484 & old eggs & $\mathrm{nc}$ & $1484+$ \\
\hline 5.5 & 468 & 631 & 339 & 1438 & old eggs & $\mathrm{nc}$ & $1438+$ \\
\hline 6.5 & ns & ns & ns & 910 & $\begin{array}{l}\text { Hatched } \\
\text { larvae }\end{array}$ & 564 & 1474 \\
\hline
\end{tabular}

12 Dec. 1969

\begin{tabular}{|c|c|c|c|c|c|c|c|}
\hline 4.5 & 186 & 249 & 222 & 657 & 01d eggs & None & 657 \\
\hline 5.0 & 329 & 373 & 158 & 860 & old eggs & None & 860 \\
\hline 5.0 & ns & ns & ns & 472 & $\begin{array}{l}\text { Hatched } \\
\text { larvae }\end{array}$ & None & 472 \\
\hline .5 & 347 & 508 & 392 & 1247 & O1d eggs & $n c$ & $1247+$ \\
\hline 5.5 & 357 & 471 & 263 & 1091 & Old eggs & $\mathrm{nc}$ & $1091+$ \\
\hline 5.5 & 280 & 313 & 183 & 776 & Old eggs & None & 776 \\
\hline 6.6 & 442 & 466 & 240 & 1128 & old eggs & None & 1128 \\
\hline
\end{tabular}

$a_{n s}$ : not separated; bnc: not counted. 


\begin{tabular}{|c|c|c|c|c|c|c|c|}
\hline \multirow[t]{2}{*}{ 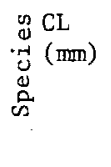 } & \multicolumn{4}{|c|}{$\begin{array}{l}\text { No. of eggs/1arvae } \\
\text { on abdomina1 ap- } \\
\text { pendages }\end{array}$} & \multirow[t]{2}{*}{$\begin{array}{l}\text { Embryonic } \\
\text { stage }\end{array}$} & \multirow{2}{*}{$\begin{array}{l}\text { No. of } \\
\text { ovar- } \\
\text { ian } \\
\text { eggsb }\end{array}$} & \multirow[t]{2}{*}{ Total } \\
\hline & 1 & 2 & 3 & Total & & & \\
\hline
\end{tabular}

\begin{tabular}{|c|c|c|c|c|c|c|c|}
\hline 5.0 & 196 & 188 & 124 & 508 & old eggs & $\mathrm{nc}$ & $508+$ \\
\hline 5.0 & 250 & 195 & 109 & 554 & 01d eggs & nc & $554+$ \\
\hline 5.0 & 153 & 195 & 135 & 483 & old eggs & $\mathrm{nc}$ & $483+$ \\
\hline $\begin{array}{l}0.0 \\
\text { s. } \\
03 \\
03\end{array}$ & ns & ns & ns & 223 & $\begin{array}{l}\text { Young } \\
\text { larvae }\end{array}$ & $n c$ & $223+$ \\
\hline$\tilde{u}^{ \pm} 5.5$ & 253 & 244 & 44 & 541 & old eggs & $\mathrm{nc}$ & $541+$ \\
\hline 离 & ns & ns & $\mathrm{ns}$ & 747 & $\begin{array}{l}\text { Hatched } \\
\text { larvae }\end{array}$ & $\mathrm{nc}$ & $747+$ \\
\hline 5.5 & 249 & 268 & 150 & 667 & 01d eggs & None & 667 \\
\hline 5.5 & ns & ns & ns & 537 & $\begin{array}{l}\text { Young } \\
\text { larvae }\end{array}$ & $\mathrm{nc}$ & $537+$ \\
\hline 6.0 & 214 & 265 & 102 & 581 & old eggs & $\mathrm{nc}$ & $581+$ \\
\hline 6.0 & ns & ns & ns & 724 & Old eggs & nc & $724+$ \\
\hline
\end{tabular}

27 Feb. 1970

\begin{tabular}{|c|c|c|c|c|c|c|c|}
\hline 3.0 & ns & ns & ns & 321 & old eggs & 247 & 568 \\
\hline 5.0 & $\mathrm{~ns}$ & $\mathrm{~ns}$ & ns & 676 & Old eggs & $\mathrm{nc}$ & $676+$ \\
\hline 5.0 & $\mathrm{~ns}$ & ns & ns & 914 & $\begin{array}{l}\text { Young } \\
\text { larvae }\end{array}$ & 579 & 1493 \\
\hline 5.5 & $\mathrm{~ns}$ & $\mathrm{~ns}$ & ns & 931 & Old eggs & 222 & 1153 \\
\hline 胥 5.5 & $\mathrm{~ns}$ & ns & ns & 875 & old eggs & 957 & 1832 \\
\hline 5.5 & $\mathrm{~ns}$ & ns & ns & 997 & old eggs & 820 & 1817 \\
\hline$\dot{3}^{5.5}$ & ns & ns & ns & 665 & old eggs & 349 & 1004 \\
\hline 5.5 & ns & ns & ns & 1054 & $\begin{array}{l}\text { Young } \\
\text { larvae }\end{array}$ & 764 & 1818 \\
\hline 6.0 & ns & ns & $\mathrm{ns}$ & 1000 & $\begin{array}{l}\text { Young } \\
\text { larvae }\end{array}$ & 457 & 1457 \\
\hline 6.0 & ns & ns & ns & 1353 & $\begin{array}{l}\text { Young } \\
\text { larvae }\end{array}$ & 862 & 2215 \\
\hline
\end{tabular}

\begin{tabular}{|c|c|c|c|c|c|c|c|}
\hline 5.0 & ns & $\mathrm{ns}$ & $\mathrm{ns}$ & 382 & old eggs & 376 & 758 \\
\hline 5.0 & ns & $\mathrm{ns}$ & $\mathrm{ns}$ & 311 & 01d eggs & 498 & 809 \\
\hline 5.0 & ns & $\mathrm{ns}$ & ns & 427 & $\begin{array}{l}\text { Hatched } \\
\text { larvae }\end{array}$ & 289 & 716 \\
\hline$\infty^{5.0}$ & $\mathrm{~ns}$ & ns & ns & 474 & $\begin{array}{l}\text { About to } \\
\text { hatch }\end{array}$ & 601 & 1075 \\
\hline 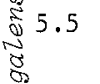 & ns & ns & ns & 457 & $\begin{array}{l}\text { Hatched } \\
\text { I arvae }\end{array}$ & 742 & 1199 \\
\hline$\sum_{0}^{\infty} 5.5$ & $\mathrm{~ns}$ & ns & ns & 689 & $\begin{array}{l}\text { Young } \\
\text { larvae }\end{array}$ & 606 & 1295 \\
\hline 85.5 & $\mathrm{~ns}$ & $\mathrm{~ns}$ & ns & 553 & $\begin{array}{l}\text { About to } \\
\text { hatch }\end{array}$ & 332 & 885 \\
\hline 5.5 & $\mathrm{~ns}$ & $\mathrm{~ns}$ & ns & 366 & $\begin{array}{l}\text { About to } \\
\text { hatch }\end{array}$ & 716 & 1082 \\
\hline 5.5 & $\mathrm{~ns}$ & $\mathrm{~ns}$ & ns & 674 & $\begin{array}{l}\text { About to } \\
\text { hatch }\end{array}$ & 490 & 1164 \\
\hline 6.5 & ns & $\mathrm{ns}$ & ns & 350 & $\begin{array}{l}\text { Hatched } \\
\text { larvae }\end{array}$ & 671 & 1021 \\
\hline
\end{tabular}

\begin{tabular}{|c|c|c|c|c|c|}
\hline \multirow{2}{*}{ 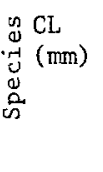 } & \multicolumn{3}{|c|}{$\begin{array}{l}\text { No. of eggs/larvae } \\
\text { on abdominal ap- } \\
\text { pendages }\end{array}$} & \multirow[t]{2}{*}{$\begin{array}{l}\text { Embryonic } \\
\text { stage }\end{array}$} & \multirow[t]{2}{*}{$\begin{array}{l}\text { No. of } \\
\text { ovar- } \\
\text { ian } \\
\text { eggsb }\end{array}$} \\
\hline & 1 & 3 & Total & & \\
\hline
\end{tabular}

20 March 1970

\begin{tabular}{|c|c|c|c|c|c|c|c|}
\hline 5.0 & ns & ns & ns & 730 & Old eggs & 748 & 1478 \\
\hline 5.0 & $\mathrm{~ns}$ & $\mathrm{~ns}$ & ns & 636 & old eggs & 209 & 845 \\
\hline 5.0 & ns & ns & ns & 950 & old eggs & 738 & 1688 \\
\hline 5.5 & ns & $\mathrm{ns}$ & $\mathrm{ns}$ & 674 & $\begin{array}{l}\text { Young } \\
\text { larvae }\end{array}$ & 790 & 1464 \\
\hline 5.5 & ns & ns & ns & 952 & 01d eggs & 1210 & 2162 \\
\hline 5.5 & ns & ns & ns & 769 & old eggs & 1101 & 1870 \\
\hline 5.5 & ns & ns & ns & 696 & old eggs & 449 & 1145 \\
\hline 5.5 & ns & ns & ns & 935 & old eggs & $22 \mathrm{I}$ & 1156 \\
\hline 6.0 & ns & ns & ns & 1282 & old eggs & None & 1282 \\
\hline 6.0 & ns & ns & ns & 1063 & $\begin{array}{l}\text { Hatched } \\
\text { larvae }\end{array}$ & 987 & 2050 \\
\hline
\end{tabular}
larvae

\begin{tabular}{|c|c|c|c|c|c|c|c|}
\hline 5.0 & ns & $\mathrm{ns}$ & ns & 655 & 01d eggs & 277 & 932 \\
\hline 5.0 & $\mathrm{~ns}$ & ns & ns & 623 & 01d eggs & 406 & 1029 \\
\hline 5.0 & $\mathrm{~ns}$ & $\mathrm{~ns}$ & ns & 483 & Old eggs & 631 & 1114 \\
\hline कू & ns & $\mathrm{ns}$ & ns & 674 & 01d eggs & 723 & 1397 \\
\hline$\widetilde{\sigma} 5.0$ & $\mathrm{~ns}$ & ns & ns & 543 & old eggs & 565 & 1108 \\
\hline 5.5 & ns & $\mathrm{ns}$ & $\mathrm{ns}$ & 770 & old eggs & 468 & 1238 \\
\hline 5.5 & ns & ns & ns & 647 & old eggs & 636 & 1283 \\
\hline 6.0 & ns & as & $\mathrm{ns}$ & 933 & OId eggs & 931 & 1864 \\
\hline 6.0 & ns & ns & ns & 868 & 01d eggs & 622 & 1490 \\
\hline 6.0 & ns & ns & ns & 963 & old eggs & 526 & 1489 \\
\hline
\end{tabular}




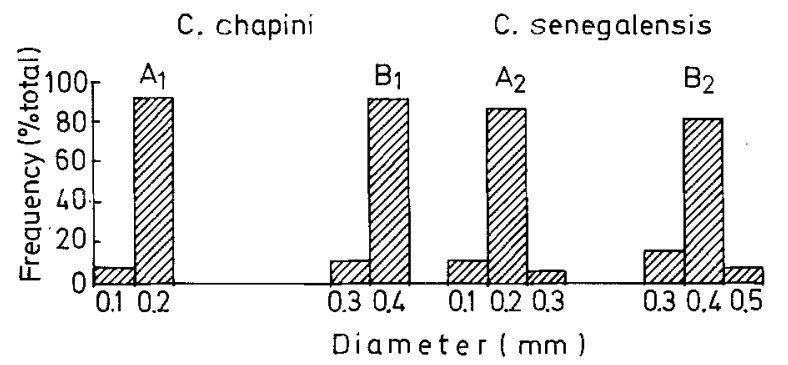

Fig. 3. Clibanamius spp. Histograms of egg sizes; $A_{1}$ and $A_{2}$ : ovarian eggs; $B_{1}$ and $B_{2}$ : pleopodal eggs

Table 5. Clibanarius chopini and C. senegalensis. Larval release

\begin{tabular}{llll}
\hline Species & $\begin{array}{l}\text { Date } \\
\text { (May, 1970) }\end{array}$ & Time (hrs) & $\begin{array}{l}\text { No. of } \\
\text { larvae } \\
\text { released }\end{array}$ \\
\hline C. chapini & 21 & 21.30 & 160 \\
& 22 & No release & - \\
23 & 13.00 & 22 \\
24 & 22.30 & 178 \\
25 & 11.00 & 16
\end{tabular}

\section{C. senegal-}

ensis

$\begin{array}{rrrr}1.21 & 21.00 & 86 \\ 22 & 11.00 & 14 \\ 23 & 22.00 & 172 \\ 21 & 20.00 & 90 \\ 24 & 13.00 & 25 \\ & 23.00 & 243\end{array}$

terns observed are thus similar to those described for other diogenids (Hazlett, 1966, 1972).

Although the mode of fertilization is not clear$1 y$ understood, presumably, as in other spermatophore-carrying decapods, fertilization is internal (see Ryan, 1967), the sperm moving along the female's oviduct and fertilizing the eggs before oviposition.

Larval Release

Some observations were made in the laboratory on larval release. Zoea larvae were released in batches of 10 to 200 . The time interval between any two successive batches can be as short as 5 min or as long as $24 \mathrm{~h}$. Individuals in each batch were carried out of the female's shell in the exhalent current which passes ventral to the head, in groups of 3,4 or 5 , at intervals of only a few seconds.

Larval release in both species occurs in the night. Observations were made on two females of clibonarius senegalensis and one of $C$. chapini over a number of days. The times of release of batches and the number of larvae released in each batch was noted (see Table 5).

Observations made on females releasing their larvae revealed that batches of larvae do not necessarily all come from the same pleopod. A female which has almost completed releasing her larvae may still have some larvae in scattered areas on all three abdominal appendages.

The exact stimuli which lead to the release of these batches of larvae, apart from a diurnal variation, are not known. This is a question of some interest since the population of larvae are all seemingly at the same stage of development and yet are released over a period of as long as 5 days.

Sex Ratio and Size Groups

In the course of the study, observations were made on the sex ratios in the population. The size groups within the populations were analysed to obtain some idea of population dynamics of the two species.

In Tables 6 and 7 , the sex ratio is recorded for each collection from different places on different dates. Although there are some cases which show a sex ratio of $1: 1$, in most cases there is a statistically significant deviation from this.

Fig. 4 presents histograms of size groups, as determined by cephalothorax length, of female and male Clibanarius chapini. There are two general features demonstrated by these histograms. First, there is a dominant size group amongst females which is not found amongst males. Second, the maximum size of the males in the samples is only slightly greater than that of the females.

In Clibcnarius senegalensis a dominant size group is present among the females, but again absent in the males (Fig. 5). The males of this species attain much greater maximum size than in $C$. chapini.

\section{Discussion}

The almost constant states of the three factors presumed to be important in breeding of tropical marine invertebrates (temperature, salinity, and food), have led to the axiom that these species breed continuously. Whether or not these factors operate exclusively or together remains a matter of controversy (see Reese, 1968). The breeding cycles of the few species investigated reveal that peaks and dips in the cycles are common (Table 8 ). Both CZibonamius chapini and $C$. senegalensis display high reproductive activity throughout the year (1owest, 40\%; highest, 90\%). An interesting feature is that both species have their lowest reproductive activities at different times of the 
year, C. chapini in November and C. senegalensis in May. These dips cannot be attributed to changes in availability of food, nor to temperature or salinity changes. Both temperature and salinity display negligible variations on the beach throughout the year and there is an overabundance of food for the hermit crabs (Ameyaw-Akumfi, in preparation). While it can be suggested that such differential dipping in the cycles may result from competition, the factors involved are not known.

Giese (1959) points out that an extended breeding season may indicate that "the individuals of a species are producing several successive broods during the year or that they are breeding asyn- chronously i.e. some are in earlier stages of maturation, some are getting ready to spawn, some are spawning and still others are already spent." Fig. 2, which illustrates the occurrence of two batches of embryonic stages, one in the ovary and one on the abdominal appendages, follows the same pattern as the plot illustrating only females in berry (Fig. 1). This suggests that there may be a production of several successive broods during a year. No studies were made on how many broods are produced by an individual female.

For a continuous breeder, one might predict continuous recruitment. Such is the case for both species in the present study. The marked differ-

Table 6. Clibanarius chapini. Sex ratio in individual samples

\begin{tabular}{|c|c|c|c|c|c|c|c|}
\hline Date & $\begin{array}{l}\text { Distance } \\
\text { from MLT } \\
\text { mark; } \\
\text { seaward } \\
\text { (m) }\end{array}$ & $\begin{array}{l}\text { No: of } \\
\text { males }\end{array}$ & $\begin{array}{l}\text { No. of } \\
\text { females }\end{array}$ & Total & $\begin{array}{l}\% \\
\text { males }\end{array}$ & $\begin{array}{l}\% \text { fe- } \\
\text { males }\end{array}$ & $\begin{array}{l}\text { Sex ratio } \\
\text { (male: } \\
\text { female) }\end{array}$ \\
\hline
\end{tabular}

1969

\begin{tabular}{|c|c|c|c|c|c|c|c|c|c|}
\hline 29 & Sept. & 10 & 14 & 16 & 30 & 46.7 & 53.3 & $1: 1.0$ & \\
\hline 4 & Oct. & 10 & 22 & 14 & 36 & 61.0 & 39.0 & $1: 0.7$ & $<0.95>0.05$ \\
\hline 24 & Oct. & $\begin{array}{r}10 \\
100\end{array}$ & $\begin{array}{l}45 \\
48\end{array}$ & $\begin{array}{r}63 \\
137\end{array}$ & $\begin{array}{l}108 \\
185\end{array}$ & $\begin{array}{l}41.7 \\
26.0\end{array}$ & $\begin{array}{l}58.3 \\
74.0\end{array}$ & $\begin{array}{l}1: 1.4 \\
1: 2.8\end{array}$ & $\begin{array}{l}<0.95>0.05 \\
<0.001\end{array}$ \\
\hline 31 & oct. & beyond 100 & 16 & 60 & 76 & 21.0 & 79.0 & $1: 3.7$ & $<0.001$ \\
\hline 12 & Nov. & $\begin{array}{r}10 \\
50 \\
100\end{array}$ & $\begin{array}{l}31 \\
33 \\
20\end{array}$ & $\begin{array}{l}71 \\
36 \\
18\end{array}$ & $\begin{array}{r}102 \\
69 \\
38\end{array}$ & $\begin{array}{l}30.5 \\
48.0 \\
52.8\end{array}$ & $\begin{array}{l}69.5 \\
52.0 \\
47.2\end{array}$ & $\begin{array}{l}1: 2.3 \\
1: 1.1 \\
1: 0.9\end{array}$ & $\begin{array}{l}<0.001 \\
<0.95>0.05 \\
<0.95>0.05\end{array}$ \\
\hline 28 & Nov. & 10 & 33 & 21 & 54 & 61.1 & 38.9 & $1: 0.6$ & $<0.95>0.05$ \\
\hline 12 & Dec. & $\begin{array}{l}10 \\
50\end{array}$ & $\begin{array}{r}100 \\
32\end{array}$ & $\begin{array}{r}234 \\
64\end{array}$ & $\begin{array}{r}334 \\
97\end{array}$ & $\begin{array}{l}30.0 \\
34.6\end{array}$ & $\begin{array}{l}70.0 \\
65.4\end{array}$ & $\begin{array}{l}1: 2.4 \\
1: 2.0\end{array}$ & $\begin{array}{l}<0.001 \\
<0.001\end{array}$ \\
\hline 1970 & & & & & & & & . & \\
\hline 23 & Jan. & $\begin{array}{r}10 \\
50 \\
100\end{array}$ & $\begin{array}{r}30 \\
178 \\
61\end{array}$ & $\begin{array}{r}78 \\
315 \\
106\end{array}$ & $\begin{array}{l}108 \\
493 \\
167\end{array}$ & $\begin{array}{l}27.8 \\
36.1 \\
36.5\end{array}$ & $\begin{array}{l}72.2 \\
63.9 \\
63.5\end{array}$ & $\begin{array}{l}1: 2.6 \\
1: 1.8 \\
1: 1.7\end{array}$ & $\begin{array}{l}<0.001 \\
<0.001 \\
<0.005\end{array}$ \\
\hline 11 & Feb. & $\begin{array}{r}10 \\
50 \\
100\end{array}$ & $\begin{array}{l}43 \\
25 \\
22\end{array}$ & $\begin{array}{r}113 \\
40 \\
54\end{array}$ & $\begin{array}{r}156 \\
65 \\
76\end{array}$ & $\begin{array}{l}27.5 \\
38.5 \\
29.0\end{array}$ & $\begin{array}{l}72.5 \\
61.6 \\
71.0\end{array}$ & $\begin{array}{l}1: 2.6 \\
1: 1.6 \\
1: 2.6\end{array}$ & $\begin{array}{l}<0.001 \\
<0.1 \\
<0.001\end{array}$ \\
\hline 27 & Feb. & $\begin{array}{r}10 \\
50 \\
100\end{array}$ & $\begin{array}{l}52 \\
27 \\
32\end{array}$ & $\begin{array}{l}76 \\
72 \\
54\end{array}$ & $\begin{array}{r}128 \\
99 \\
86\end{array}$ & $\begin{array}{l}40.6 \\
27.3 \\
37.2\end{array}$ & $\begin{array}{l}59.4 \\
72.7 \\
62.8\end{array}$ & $\begin{array}{l}1: 1.5 \\
1: 2.6 \\
1: 1.7\end{array}$ & $\begin{array}{l}<0.05 \\
<0.005 \\
<0.05\end{array}$ \\
\hline 20 & March & $\begin{array}{r}10 \\
50 \\
100\end{array}$ & $\begin{array}{l}30 \\
46 \\
15\end{array}$ & $\begin{array}{r}121 \\
74 \\
42\end{array}$ & $\begin{array}{r}151 \\
120 \\
57\end{array}$ & $\begin{array}{l}19.9 \\
32.3 \\
26.3\end{array}$ & $\begin{array}{l}80.1 \\
61.7 \\
73.7\end{array}$ & $\begin{array}{l}1: 4.0 \\
1: 1.6 \\
1: 2.5\end{array}$ & $\begin{array}{l}<0.001 \\
<0.05 \\
<0.025\end{array}$ \\
\hline 20 & April & $\begin{array}{l}10 \\
50\end{array}$ & $\begin{array}{l}68 \\
25\end{array}$ & $\begin{array}{r}136 \\
70\end{array}$ & $\begin{array}{r}204 \\
95\end{array}$ & $\begin{array}{l}33.3 \\
26.3\end{array}$ & $\begin{array}{l}66.7 \\
73.7\end{array}$ & $\begin{array}{l}1: 2.0 \\
1: 2.8\end{array}$ & $\begin{array}{l}<0.005 \\
<0.005\end{array}$ \\
\hline 23 & May & $\begin{array}{l}10 \\
50\end{array}$ & $\begin{array}{l}12 \\
33\end{array}$ & $\begin{array}{r}29 \\
132\end{array}$ & $\begin{array}{r}41 \\
165\end{array}$ & $\begin{array}{l}29.3 \\
20.0\end{array}$ & $\begin{array}{l}70.7 \\
80.0\end{array}$ & $\begin{array}{l}1: 2.3 \\
1: 4.0\end{array}$ & $\begin{array}{l}<0.005 \\
<0.005\end{array}$ \\
\hline 20 & June & 10 & 49 & 152 & 201 & 24.4 & 75.6 & $1: 3.0$ & $<0.005$ \\
\hline
\end{tabular}


ences in size patterns of males and females raise the question of their significance. One interpretation, that there was a highly successful "class" of females which dominate all samples seems untenable; samples had been collected at monthly intervals, and the modal size of the females remained effectively constant over a period of 9 months (Figs. 4 and 5). Although no direct information upon growth rates is available, it seems improbable that so stable a situation would have persisted for so long a period.

The data are more easily interpreted as indicating that the growth rate of the female slows mar- kedly on attainment of sexual maturity. The inhibitory effect of egg-carrying on moulting in the female has been discussed by Passano (1960). It is possible that there is a direct selection for a check in growth rate independent of any nutritional drain. Unlike the males, females have to accomodate not only themselves, but also their brood within the confines of a gastropod shell and, should the female continue to grow beyond sexual maturity, she might be unable to find a suitable shell to accomodate herself and her brood. In fact, a survey of available empty shells of the species used by these crabs provided no evidence for a re-

Table 7. Clibanarius senegalensis. Sex ratio in individual samples

\begin{tabular}{|c|c|c|c|c|c|c|c|}
\hline Date & $\begin{array}{l}\text { Distance } \\
\text { from MLT } \\
\text { mark; } \\
\text { seaward }\end{array}$ & $\begin{array}{l}\text { No. of } \\
\text { males }\end{array}$ & $\begin{array}{l}\text { No. of } \\
\text { females }\end{array}$ & Total & $\begin{array}{l}\% \\
\text { males }\end{array}$ & $\begin{array}{l}\% \text { fe- } \\
\text { males }\end{array}$ & $\begin{array}{l}\text { Sex ratio } \\
\text { (male: } \\
\text { female) }\end{array}$ \\
\hline
\end{tabular}

(m)

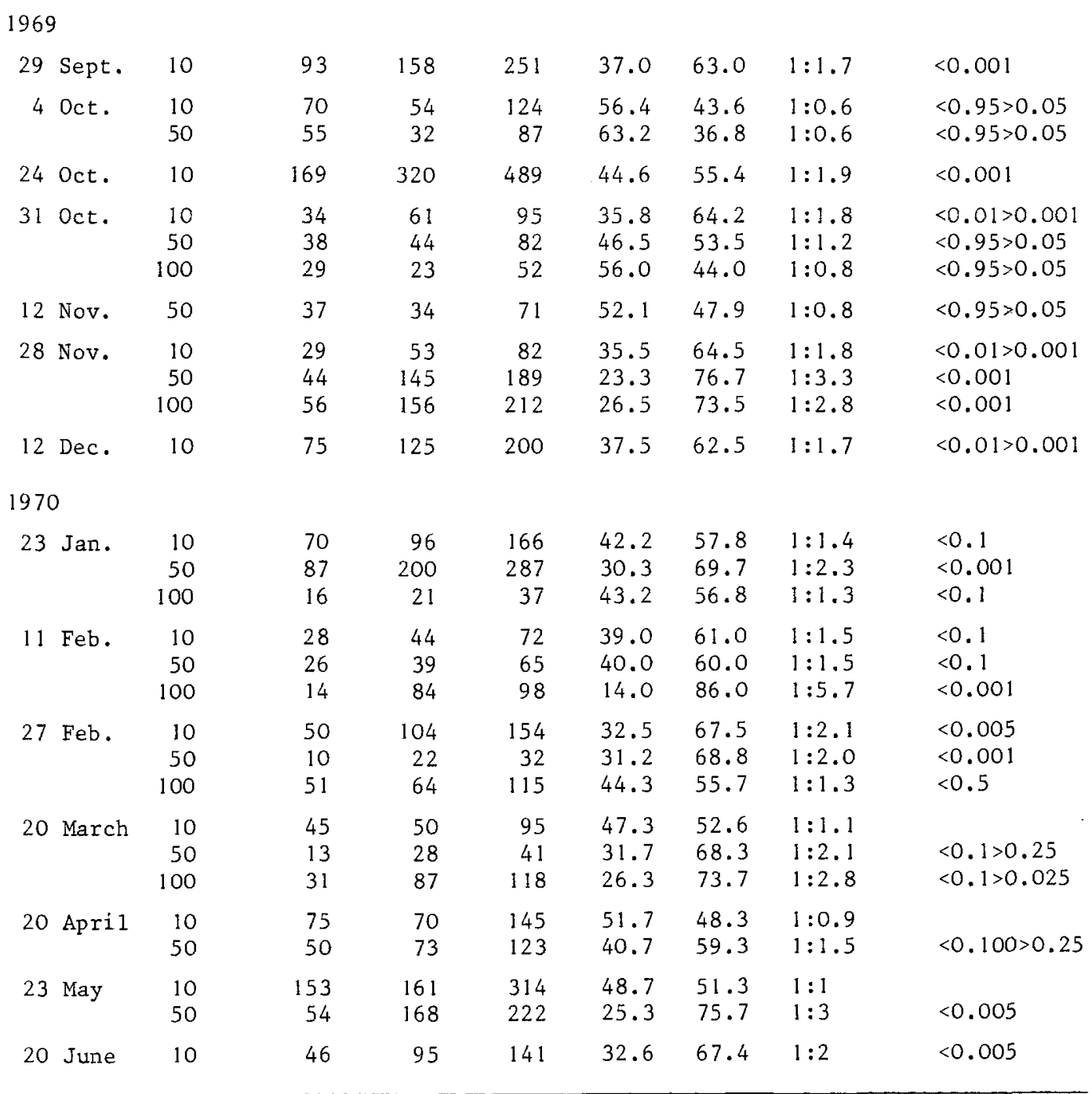



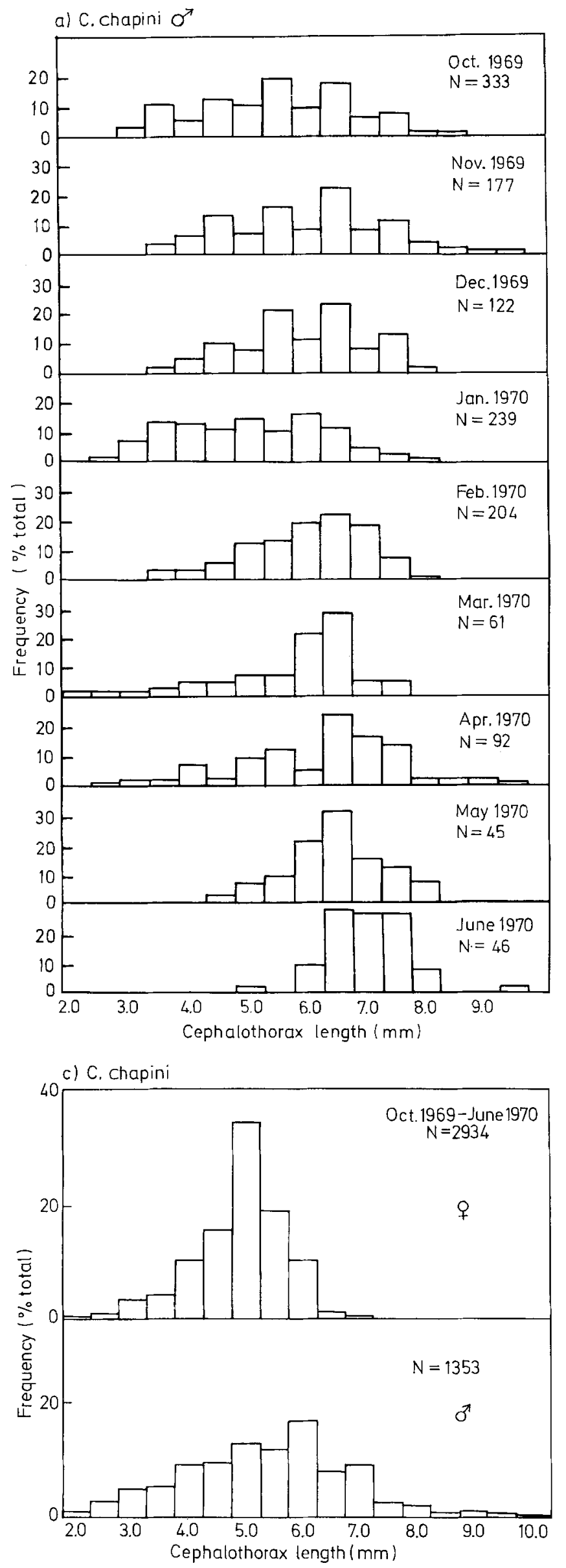

b) C. Chapini +

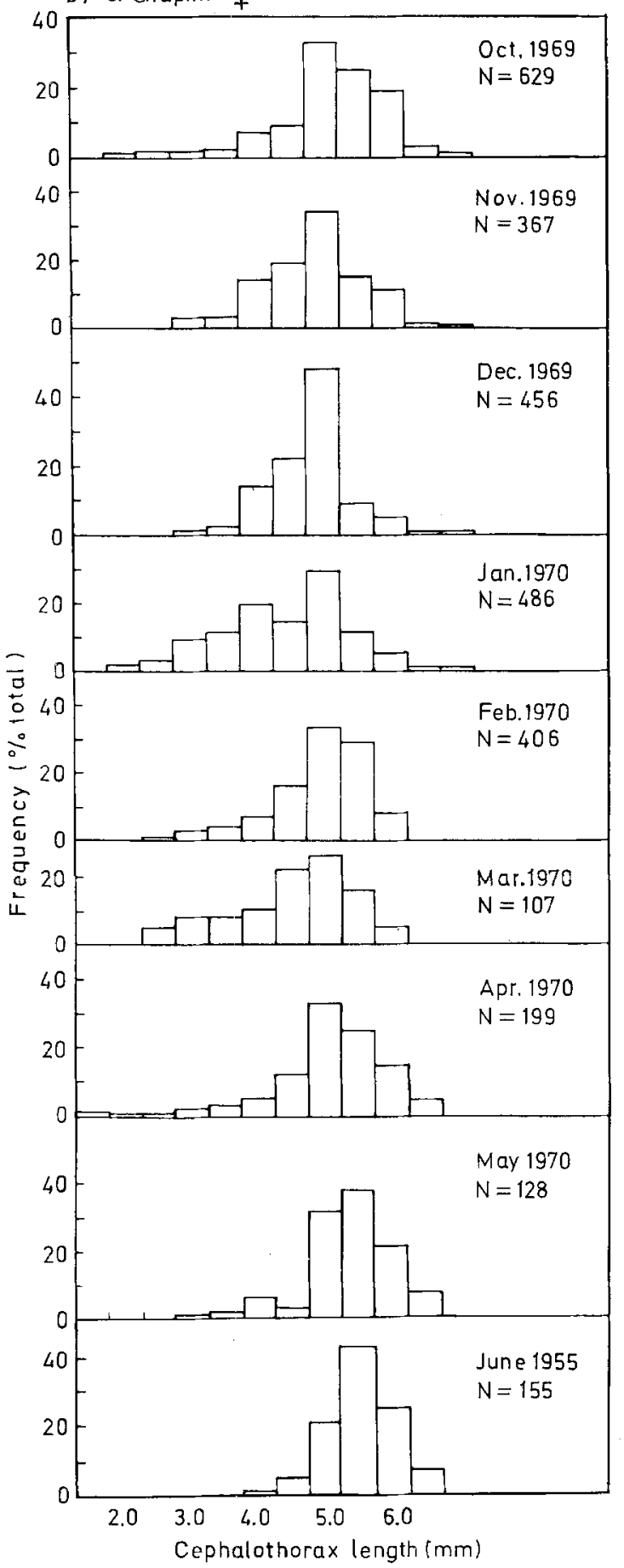



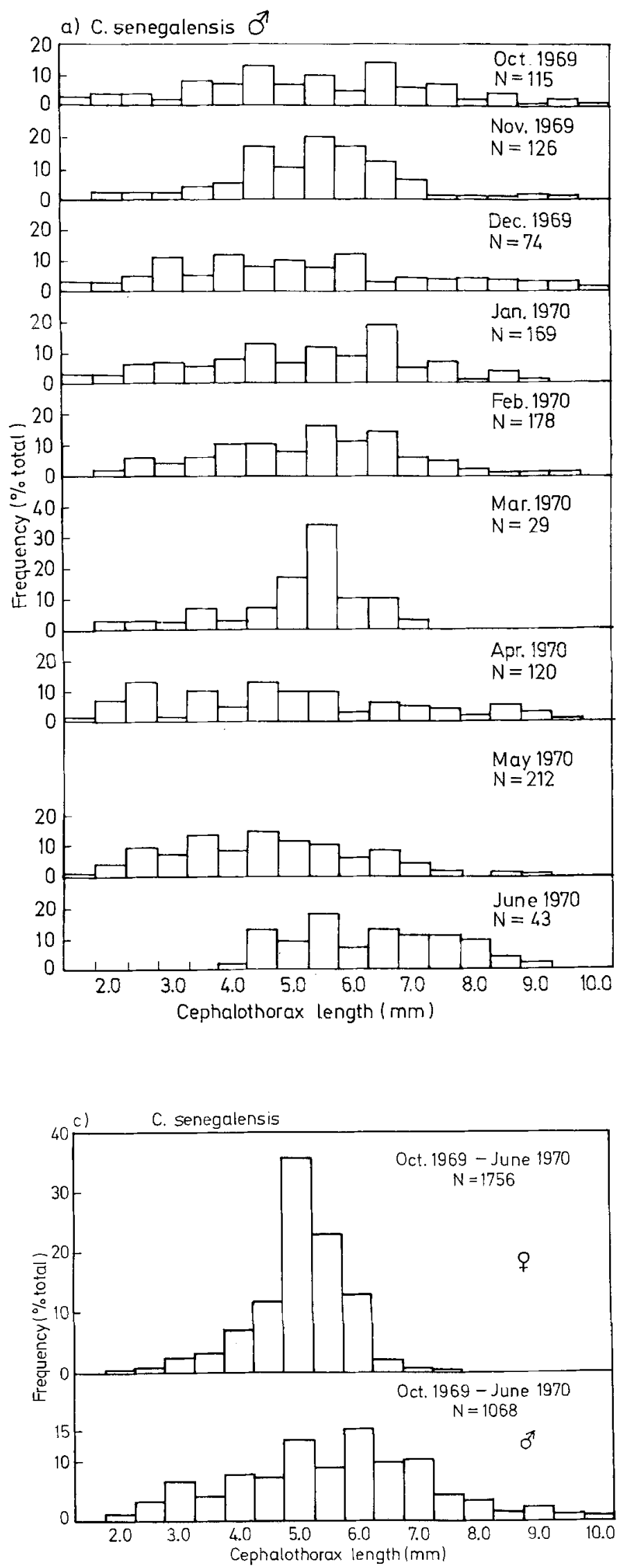

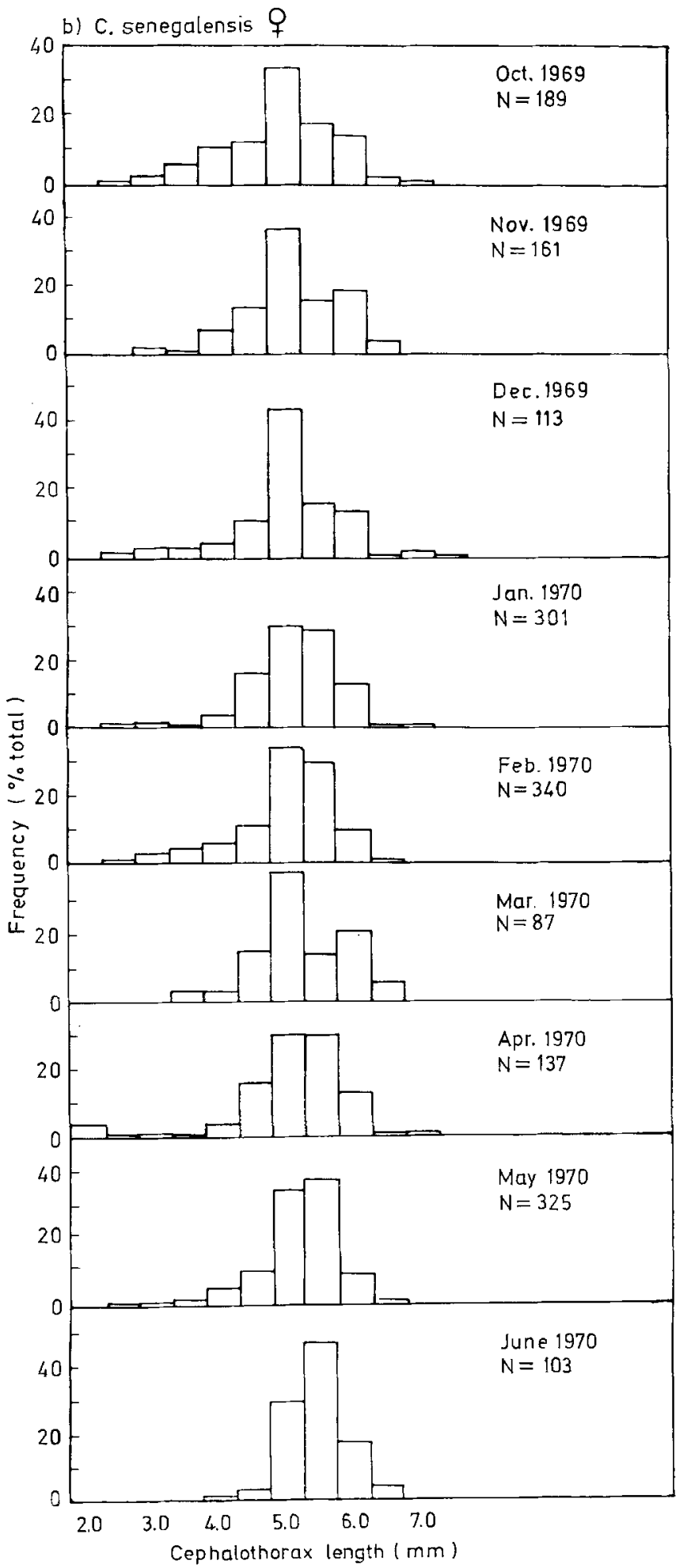

Fig. 5. Clibanamius senegalensis. Histograms of size groups of males (a) and females (b) from monthly samples, and combined males and females (c) for whole 9-month study 
serve of large, unused shells. In other words, the adults attain as great an adult size as can be tolerated in the snail shells which they occupy (Markham, 1968) and, in these circumstances, we might indeed expect females to be smaller than males. This, however, does not explain why both male and female Clibanarius chapini grow to almost the same size.

Finally, a word about the time of larval release. This occurred in the night in both species in the laboratory, and most probably also occurs in nature. Fabre Domergue and Bietrix (1903) described hatching in the lobster Homams gammarus (L.) (=H. vulgaris Milne Edwards), stating that it occurred during the early hours of the night. Fullarton (1896), in the same species, described the total time required for hatching of a brood to vary from 3 to 5 weeks or even longer. Recently, Ennis (1973) described such nocturnal larval release in H. gammarus lasting 2 to 6 weeks. In the sand lobster ScyZzarus omericanus, Robertson (1968) also reported nocturnal larval release. Locomotor activity studies on both Clibanarius species revealed that both $C$. chapini and $C$. senegazensis are nocturnal, although the former also showed considerable activity in the day time (AmeyawAkumfi, unpublished data). Since larvae are released in the expiratory current, it is reasonable to assume that the larval releasing mechanism might be contingent to the general activity of the adult female.

Acknowledgements. This study forms part of work performed for the M.Sc. degree at the University of Ghana, Legon, Ghana. My sincere thanks to the University for its support and to members of my research committee, Dr. D.W. Ewer, Dr. M. Edmunds and Mr. W. Popple, all formerly of the University of Ghana. I also thank Dr. B.A. Hazlett of the University of Michigan, Ann Arbor, Michigan, USA, for his criticism of the manuscript.

Table 8. Recorded breeding activities of some tropical invertebrates

\begin{tabular}{|c|c|c|c|c|c|}
\hline Species & $\begin{array}{l}\text { Reproduc- } \\
\text { tive pat- } \\
\text { tern }\end{array}$ & $\begin{array}{l}\text { Reproduc- } \\
\text { tive peak }\end{array}$ & $\begin{array}{l}\text { Lowest re- } \\
\text { productive } \\
\text { activity } \\
\text { level }\end{array}$ & Locality & Source \\
\hline $\begin{array}{l}\text { Pugettia } \\
\text { producta }\end{array}$ & Continuous & \multicolumn{2}{|c|}{$\begin{array}{l}\text { Fairly constant level } \\
\text { throughout year }\end{array}$} & $\begin{array}{l}\text { Central Cali- } \\
\text { fornian coast } \\
\left(36030^{\prime} \mathrm{N}\right)\end{array}$ & $\begin{array}{l}\text { Boolootian } \\
\text { etat. } \\
(1959)\end{array}$ \\
\hline $\begin{array}{l}\text { Petrolisthes } \\
\text { cinctipes }\end{array}$ & Continuous & Jan.-March & Oct.-Nov. & $\begin{array}{l}\text { Central Cali- } \\
\text { fornian coast } \\
\left(36^{\circ} 30^{\prime} \mathrm{N}\right)\end{array}$ & $\begin{array}{l}\text { Boolootian } \\
\text { et al. } \\
(1959)\end{array}$ \\
\hline $\begin{array}{l}\text { Hemigrapsus } \\
\text { nudus }\end{array}$ & Continuous & Nov.-Jan. & $\begin{array}{l}\text { March- } \\
\text { Sept. }\end{array}$ & $\begin{array}{l}\text { Central Cali- } \\
\text { fornian coast } \\
\left(36^{\circ} 30^{\prime} \mathrm{N}\right)\end{array}$ & $\begin{array}{l}\text { Boolootian } \\
\text { et al. } \\
(1959)\end{array}$ \\
\hline $\begin{array}{l}\text { Pachygrapsus } \\
\text { crassipes }\end{array}$ & $\begin{array}{l}\text { Seasonal } \\
\text { (March- } \\
\text { Aug.) }\end{array}$ & June-July & $\begin{array}{l}\text { March, } \\
\text { April and } \\
\text { August }\end{array}$ & $\begin{array}{l}\text { Central Cali- } \\
\text { fornian coast } \\
\left(36^{\circ} 30^{\prime} \mathrm{N}\right)\end{array}$ & $\begin{array}{l}\text { Boolootian } \\
\text { et al. } \\
(1959)\end{array}$ \\
\hline $\begin{array}{l}\text { Ememita } \\
\text { analoga }\end{array}$ & $\begin{array}{l}\text { Seasonal } \\
\text { (April- } \\
\text { Oct.) }\end{array}$ & June-July & $\begin{array}{l}\text { April-May, } \\
\text { Sept.-Oct. }\end{array}$ & $\begin{array}{l}\text { Central Cali- } \\
\text { fornian coast } \\
\left(36^{\circ} 30^{\circ} \mathrm{N}\right)\end{array}$ & $\begin{array}{l}\text { Boolootian } \\
\text { et al. } \\
(1959)\end{array}$ \\
\hline $\begin{array}{l}\text { Mysidium } \\
\text { columbiae }\end{array}$ & Continuous & $\begin{array}{l}\text { Nov. }(1960) \text {, } \\
\text { Aug. }(1961)\end{array}$ & $\begin{array}{l}\operatorname{Jan} .(1960) \text {, } \\
\operatorname{Oct} \cdot(1961)\end{array}$ & $\begin{array}{l}\text { Jamaica } \\
(180 \mathrm{~N})\end{array}$ & $\begin{array}{l}\text { Goodbody } \\
(1965)\end{array}$ \\
\hline $\begin{array}{l}\text { Emerita por- } \\
\text { tomicensis }\end{array}$ & Continuous & $\begin{array}{l}\text { Feb., May- } \\
\text { Sept. }\end{array}$ & $\begin{array}{l}\text { April, } \\
\text { Oct.-Dec. }\end{array}$ & $\begin{array}{l}\text { Jamaica } \\
\left(18^{\circ} \mathrm{N}\right)\end{array}$ & $\begin{array}{l}\text { Goodbody } \\
(1965)\end{array}$ \\
\hline $\begin{array}{l}\text { Petrolisthes } \\
\text { elongatus }\end{array}$ & Continuous & $\begin{array}{l}\text { Winter and } \\
\text { early } \\
\text { spring }\end{array}$ & $\begin{array}{l}\text { Fal1 and } \\
\text { summer }\end{array}$ & $\begin{array}{l}\text { Hauraki Gulf, } \\
\text { New Zealand } \\
\left(35^{\circ} \mathrm{S}\right)\end{array}$ & $\begin{array}{l}\text { Greenwood } \\
(1965)\end{array}$ \\
\hline $\begin{array}{l}\text { Calcinus } \\
\text { Zaevimanus }\end{array}$ & Continuous & May-Aug. & Nov.-Dec. & $\begin{array}{l}\text { Kaneoho, Oahu } \\
\text { Bay, Hawaii } \\
\left(21^{\circ} 30^{\prime} \mathrm{N}\right)\end{array}$ & $\begin{array}{l}\text { Reese } \\
(1968)\end{array}$ \\
\hline $\begin{array}{l}\text { Calcinus } \\
\text { Zatens }\end{array}$ & Continuous & $\begin{array}{l}\text { Feb.- } \\
\text { March, } \\
\text { Aug. }\end{array}$ & Oct.-Dec. & $\begin{array}{l}\text { Kaneoho, Oahu } \\
\text { Bay, Hawaii } \\
\left(21^{\circ} 30^{\prime} N\right)\end{array}$ & $\begin{array}{l}\text { Reese } \\
(1968)\end{array}$ \\
\hline $\begin{array}{l}\text { Clibanarius } \\
\text { zebra }\end{array}$ & Continuous & $\begin{array}{l}\text { Feb.- } \\
\text { April, } \\
\text { Aug. }\end{array}$ & $\begin{array}{l}\text { May-July, } \\
\text { Sept.-Nov. }\end{array}$ & $\begin{array}{l}\text { Kaneoho, Oahu } \\
\text { Bay, Hawaii } \\
\left(21^{\circ} 30^{\prime} \mathrm{N}\right)\end{array}$ & $\begin{array}{l}\text { Reese } \\
(1968)\end{array}$ \\
\hline
\end{tabular}




\section{Iiterature Cited}

Boolootian, R.A., A.C. Giese, A. Farmanfarmian and J. Tucker: Reproductive cycles of five west coast crabs. Physiol. Zoö1. 32, 213-220 (1959)

Buchanan, D.V. and R.E. Millemann: The prezoeal stage of the Dungeness crab, Cancer magister Dana. Biol. Bull. mar. biol. Lab., Woods Hole $137,250-255$ (1969)

Ennis, G.P.: Endogenous rhythmicity associated with larval hatching in the lobster Homarus gammarus. J. mar. biol. Ass. U.K. 53, 531-538 (1973)

Fabre-Domergue et E. Bietrix: Le mecanisme de 1 'emission des larves chez la femelle du homrad européen. C. r. hebd. Séanc. Acad. Sci., Paris $136,1408-1409$ (1903)

Fullarton, J.H.: The European lobster: breeding and development. Rep. Fishery Bd Scot1. 14, 186222 (1896)

Giese, A.C.: Comparative physiology: annual reproductive cycles of marine invertebrates. A. Rev. Physiol. 21, 547-576 (1959)

Goodbody, I.: Continuous breeding in populations of two tropical crustaceans Mysidium columbiae (Zimmer) and Emerita portoricensis Schmitt. Ecology 46, 195-197 (1965)

Greenwood, J.G.: The larval development of Petrolisthes elongatus (H. Milne-Edwards) and Petrolisthes novaezelandiae Filhol (Anomura, Porcellanidae) with notes on breeding. Crustaceana 8 , 285-307 (1965)

Gunter, G.: Temperature. In: Treatise on marine ecology and paleoecology. Vo1. 1. Ecology, pp 159-184. Ed. by J.W. Hedgpeth. New York: Geological Society of America 1957

Hazlett, B.A.: Social behaviour of the Paguridae and Diogenidae of Curaçao. Stud. Fauna Curaçao $23,1-143$ (1966)

- Tactile stimuli in the social behaviour of Pagums bemhardus (Decapoda, Paguridae). Behaviour $36,20-48$ (1970)

- Shell fighting and social behaviour in the hermit crab genera Paguristes and Calcinus with comments on Fagums. Bul1. mar. Sci. 22, 806823 (1972)

Herrnkind, W.: The breeding of Uca pugizator (Bosc) and the mass rearing of the larvae with comments on the behaviour of the larval and early crab stages (Brachyura, Ocypodidae). Crustaceana (Supp1.) 2, 214-224 (1968)

Knight, M.D.: The larval development of the sand crab Emerita rathbunae Schmitt (Decapoda, Hippidae). Pacif. Sci. 27, 58-76 (1967)
Knowlton, R.E.: Effects of some environmental factors on the larval development of Palaemonetes vulgaris (Say). J. Elisha Mitchell scient. Soc. 81, p. 87 (1965)

Little, G.: Induced winter breeding and larval development in the shrimp Palaemonetes pugio Holthuis (Caridae, Palaemonidae). Crustaceana (Supp1.) $2,19-26$ (1968)

- The larval development of the shrimp Palaemon macrodactylus Rathbun, reared in the laboratory, and the effect of eyestalk extirpation on development. Crustaceana 17, 69-87 (1969)

Lewis, J.B.: The fauna of the rocky shores of Barbados, West Indies. Can. J. Zoo1. 38, 391-435 (1960)

Markham, J.C.: Notes on growth patterns and shell utilization of the hermit crab Pagums bermhardus (L.) Ophelia 5, 189-205 (1968)

Moore, H.B.: Marine ecology, 493 pp. New York: John Wiley \& Sons 1958

Passano, L.M.: Molting and its control. In: The physiology of Crustacea, Vol. 1. pp 473-536. Ed. by T.H. Waterman. New York: Academic Press 1960

Reese, E.: Annual breeding seasons of three sympatric species of tropical intertidal hermit crabs, with a discussion of factors controlling breeding. J, exp. mar. Biol. Ecol. 2, 308-318 (1968)

Robertson, P.B.: The complete larval development of the sand lobster Scyzzarus americanus (Smith) (Decapoda, Scyllaridae) in the laboratory, with notes on larvae from the plankton. Bul1. mar. Sci. 18, 294-342 (1968)

Ryan, E.P.: Structure and function of the reproductive system in the crab Portums sanguinoZentus. Proc. Symp. Crustacea (Mar. biol. Ass., India) 2, 506-521 (1967)

Sandoz, M. and R. Rogers: The effect of environmental factors on hatching, moulting and survival of zoea larvae of the blue crab, Calzinectes sapidus Rathbun. Ecology 25, 216-228 (1944)

Thorson, G.: Reproduction and the larval development of Danish marine bottom invertebrates. Meddr Kommn Danm. Fisk.-og Havunders. (Ser. Plankton) 4, 1-523 (1946)

- Reproductive and larval ecology of marine bottom invertebrates. Bio1. Rev. 25, 1-45 (1950)

Mr. C. Ameyaw-Akumfi Department of Zoology University of Michigan Ann Arbor, Michigan 48104 USA

Date of final manuscript acceptance: November 4, 1974. Communicated by 0 . Kinne, Hamburg 